\title{
The role of the gut as a primary lymphoid organ: CD8 a intraepithelial T lymphocytes in euthymic mice derive from very immature CD44+ thymocyte precursors
}

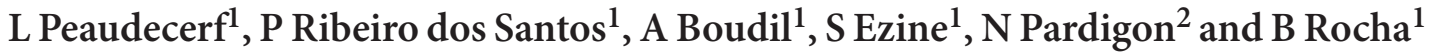

Intestinal CD8 $\alpha \alpha$ intraepithelial T lymphocytes (T-IELs) have a key role in mucosal immunity and, unlike other T cells, were proposed to differentiate locally. In apparent contradiction, these cells were also shown to originate from a wave of thymus migrants colonizing the gut in the first 3 weeks after birth. We here identify previously uncharacterized very immature $\mathrm{CD} 4-\mathrm{CD} 8-\mathrm{CD} 3-\mathrm{CD} 44^{+} \mathrm{CD} 25^{\text {int }}$ thymocytes, which have not yet rearranged their T-cell antigen receptor (TCR), as having the capacity to leave the thymus, migrate to the blood, colonize the gut, and reconstitute CD8 $\alpha \alpha$ T-IEL, and show that this cell set is fully responsible for the generation of the CD8 $\alpha \alpha$ T-IEL pool. Thus, although the thymus may be fundamental for efficient T-cell commitment, CD $8 \alpha \alpha$ T-IEL' complete TCR rearrangements and TCR- $\alpha \beta / \gamma \delta$ lineage commitment must occur in the gut. These results demonstrate a major role of the gut environment as a primary lymphoid organ.

\section{INTRODUCTION}

Besides conventional T-cell antigen receptor (TCR)- $\alpha \beta^{+}$ $\mathrm{CD} 4^{+}$or $\mathrm{CD} 8 \alpha \beta^{+}$cells, the gut epithelium harbors a very abundant and unique $\mathrm{T}$ lymphocyte population that expresses the CD8 $\alpha \alpha$ homodimeric form of the CD8 coreceptor (CD8 $\alpha \alpha$ T-IEL), and has a fundamental role in maintaining the integrity of the gut wall. ${ }^{1}$ This cell set is enriched in TCR- $\gamma \delta^{+}$ cells, and also harbors an agonist selected TCR- $\alpha \beta^{+}$set. ${ }^{1}$ As in the absence of a thymus, these cells develop from Lineage ${ }^{-}$ $\left(\mathrm{Lin}^{-}\right) \mathrm{c}$-kit ${ }^{+}$interleukin (IL)-7R ${ }^{+}$precursors found within small clusters of hematopoietic cells located in the lamina propria (LP) in-between the gut crypts, the gut cryptopatches $(\mathrm{CP}),{ }^{2,3}$ it was suggested that CD $8 \alpha \alpha$ T-IEL were generated extrathymically. However, the relative role of such an extrathymic differentiation pathway in normal conditions has been questioned, as, in euthymic mice, CD8 $\alpha \alpha$ T-IEL in general and TCR- $\alpha \beta^{+}$cells in particular are much more abundant and have different characteristics; moreover, both TCR- $\alpha \beta^{+}$ and TCR- $\gamma \delta^{+}$cells were shown to derive from a wave of thymus migrants repopulating the gut in the first 3 weeks after birth. ${ }^{4,5}$
It was proposed that CD8 $\alpha \alpha$ T-IELs were the progeny of relatively mature thymocyte sets already expressing a rearranged TCR, thus excluding a T-cell differentiation process occurring in the gut in normal conditions. On the basis of the experiments conducted in mice expressing transgenic TCR specific of the HY antigen, it was proposed that TCR- $\alpha \beta^{+}$CD $8 \alpha \alpha$ T-IEL derive from a minority $\mathrm{CD} 4^{+} \mathrm{CD} 8 \alpha \beta^{+}$double-positive (DP) population co-expressing the characteristic CD $8 \alpha \alpha$ homodimers of CD8 $\alpha \alpha$ T-IEL. These cells would be identified by a specific ability of CD8 $8 \alpha$ to bind tetramers of the thymus leukemia antigen (TL-tetramers (TL-tet)), would undergo agonist selection in the cortex, and differentiate into NK1.1 ${ }^{-}$TCR$\alpha \beta^{+} \mathrm{CD} 4^{-} \mathrm{CD} 8^{-}(\mathrm{DN})$ cells that would reconstitute TCR- $\alpha \beta^{+}$ CD8 $\alpha \alpha$ T-IEL. ${ }^{6}$ Gene mapping of ROR $\gamma$ t-expressing cells and their progeny also proposed a DP origin, ${ }^{7}$ but these notions are somehow controversial, as other reports using either anti-HY TCR-Tg mice ${ }^{8}$ or ROR $\gamma$ t gene mapping ${ }^{9,10}$ gave opposite results (SOM-1). Other data supported that the thymus contribution would rather involve immature thymocyte populations, indicating that a $\mathrm{T}$-cell differentiation process could occur in the gut environment of euthymic mice. It was shown that grafts

${ }^{1}$ INSERM, U1020, Medical Faculty Descartes Paris V, Paris, France. ${ }^{2}$ Unité des interactions Flavivirus-Hôtes, Institut Pasteur, Paris, France. Correspondence: B Rocha (benedita.rocha@inserm.fr) 
from $\mathrm{CD}^{-} \mathrm{CD}^{-} \mathrm{CD}^{-}$(triple negative (TN)) thymocytes ${ }^{11}$ or Lin ${ }^{-} \mathrm{CP}$ cells from euthymic mice ${ }^{12}$ reconstitute "euthymiclike" CD8 $\alpha \alpha$ T-IEL and that $\mathrm{Lin}^{-}$cells isolated from the CP or the epithelium show clear evidence of an ongoing T-cell differentiation in both mouse and man. ${ }^{9,11-15}$ However, limitations of data obtained from transfer experiments, in which cell sets are injected artificially into the blood, and the complexity of the TN populations, in which many T-cell differentiation events could have already taken place, do not allow identifying the relative contribution of thymic and extrathymic T-cell differentiation events in CD8 $\alpha \alpha$ T-IEL generation. Moreover, in normal mice, the majority of CD $8 \alpha \alpha$ T-IEL could derive from mature thymocyte sets and the contribution of TN populations could be minor.

We here used several complementary approaches to identify which thymocyte sets were able to leave the thymus, colonize the gut, and generate CD8 $\alpha \alpha$ T-IEL. We found that a previously uncharacterized TN sub-population that had not yet rearranged the TCR was the thymus precursor of the CD8 $\alpha \alpha$ T-IEL. Therefore, the gut environment should guarantee both TCR rearrangements and TCR- $\alpha \beta / \gamma \delta$ lineage commitment of CD8 $\alpha \alpha$ T-IEL. In this context, it must be considered as a primary lymphoid organ responsible for the generation of this major IEL subset in normal conditions in euthymic mice.

\section{RESULTS \\ Identification of the TN subsets capable of thymus egress and gut reconstitution}

To characterize which TN subsets are able to generate CD $8 \alpha \alpha$ T-IEL, we initially sorted TN subsets based on their expression of CD44 and CD25 and injected them intravenously into nude mice. Only CD $44^{+} \mathrm{TN}$ thymocytes were able to generate "euthymic-like" CD8 $\alpha \alpha$ T-IEL (SOM-2, Supplementary Figure S1 online), but in these experimental conditions TN cells gain the blood stream artificially. To study if any immature subset is able to exit an intact thymus, CD $45.1^{+} \mathrm{Rag}-2 / \gamma_{\mathrm{c}}{ }^{-1-}$ mice were transplanted with CD $45.2^{+}$neonatal thymi and studied shortly after grafting (SOM-3). This combination was selected as adapted to the characteristics of gut colonization. Thus, as CD8 $\alpha \alpha$ T-IELs are generated after birth, we used neonatal rather than fetal thymi in these experiments. Rag- $2 / \gamma_{c}{ }^{-1-}$ mice were used as hosts as they are devoid of $\mathrm{CP}$, allowing the colonization of the gut by putative precursors. We found that besides mature $\mathrm{T}$ cells the thymus transplants also exported $\mathrm{Lin}^{-} \mathrm{CD} 45.2^{+}$cells into the blood (Figure 1a, Supplementary Figure S2A online). All migrants expressed CD44, but the bulk of thymus export appeared to be constituted by a previously non-identified cell type with a phenotype corresponding to the transition between the $\mathrm{TN} 1$ and the $\mathrm{TN} 2$ thymocyte sets. Indeed, early TN1 thymocyte progenitors (ETP) are $\mathrm{CD} 44^{+} \mathrm{CD} 25^{-}$Thy $1^{-}$IL-7 ${ }^{-} \alpha^{-} \mathrm{Sca}-{ }^{+}{ }^{+}$- $-\mathrm{kit}^{+}$, and during their differentiation into TN2 they acquire the expression of CD25, Thy1, and IL-7R, whereas Sca-1 expression is downregulated $^{16,17}$ (Supplementary Figure S3 online). Although rare migrants with the phenotype of ETP or TN2 could be visualized in the blood, the vast majority of $\mathrm{Lin}^{-} \mathrm{CD} 45.2^{+}$was c-kit ${ }^{+}$ IL-7R $\alpha^{\text {int/- }}$ and expressed intermediary levels of CD25, Sca- 1 and
Thy1 as compared to TN1 and TN2 (Figure 1a, Supplementary Figure S3 online). CD $44^{+}$thymus migrants also colonized the gut LP (Figure 1a), where they have the unique Sca- $1^{\text {high }}$, $\mathrm{CD} 25^{-} /$low $_{\mathrm{C}-\mathrm{kit}}{ }^{\text {high }}$ phenotype characteristic of $\mathrm{Lin}^{-} \mathrm{CP}$ cells. ${ }^{12}$ These results indicate that thymus migrants change their phenotype in the LP environment, as their compound phenotype does not correspond to any thymocyte set. Moreover, such high c-kit expression level is not found in the thymus, but is characteristic of the gut CP cells. ${ }^{12,18}$ Lin $^{-}$migrants of thymus origin expressed rag1 and a smaller number expressed $c d 3 \varepsilon$ (Figure $\mathbf{1 b}$ ), as characteristic of early TN precursors.

The presence of a dominant TN1 to TN2 transitional population in the blood of transplanted mice indicated a selected export, i.e., that neonatal thymi were not passively leaky (SOM-4, Supplementary Figure S2B online). However, as thymus transplantation also involves an artificial manipulation and it is generally believed that the thymus only exports mature $\mathrm{T}$ cells, we next studied all thymocyte populations directly including the TN1 to TN2 transitional set, to investigate their potential to exit the thymus and migrate to the gut. Thymus egress requires the expression of $\mathrm{S}_{1} \mathrm{P}_{1}$, a receptor of sphingosine-1-phosphate (S1P) ${ }_{1}^{19}$ whereas migration to the gut requires the expression of the $\alpha_{4} \beta_{7}$ integrin. ${ }^{20,21}$ We found that besides mature thymocytes, only $\mathrm{CD} 44^{+} \mathrm{TN}$ cells expressed these molecules, but expression frequencies were maximal in the TN1 to TN2 transition. Indeed, although $\sim 20 \%$ of TN1 and TN2 cells expressed $s 1 p 1$, the s1p1 expression frequencies of the TN1 to TN2 intermediate populations were much higher, approaching those found in mature $\mathrm{TCR}^{+} \mathrm{CD} 4^{+} \mathrm{CD}^{-}$thymocytes. Neither TN3, TN4 nor DP cells expressed $\operatorname{sip} 1$ (Figure 2a). Similarly, we found an important upregulation of $\alpha_{4} \beta_{7}$ in the TN1 to TN2 transition, whereas $\alpha_{4} \beta_{7}$ expression was lost in the TN2 to TN3 transition: double labeling with CD44 and $\alpha_{4} \beta_{7}$ of TN2 + TN3 sets showed that the loss of CD44 directly correlated to $\alpha_{4} \beta_{7}$ decline (Figure $2 \mathbf{b}$ ). Neither TN3, TN4 nor DP cells expressed $\alpha_{4} \beta_{7}$ (not shown). We conclude that besides mature T cells, only CD $44^{+} \mathrm{TN}$ populations harbor a significant fraction of cells that can egress the thymus and migrate to the gut. Both the direct study of thymocyte population properties and the characterization of TN migrants in the blood after thymus transplantation indicate that the bulk of TN egress should occur during the TN1 to TN2 transition. It must be noted that the TN1 to TN2 transition population is particularly abundant during the perinatal period when the gut colonization is known to occur (Figure 2b).

\section{Characterization of more mature CD8 $\alpha \alpha$ T-IEL precursors in normal mice}

The repertoire of TCR- $\alpha \beta$ CD $8 \alpha \alpha$ T-IEL is not deleted by the presence of self-antigens, but it is rather agonist selected. ${ }^{22}$ Studies mostly performed in TCR-Tg mice postulated that CD8 $\alpha \propto$ T-IEL rather than being generated from immature thymocyte precursors would issue from a DP population that, alike these IEL, would also express CD8 $\alpha \alpha{ }^{6}{ }^{6}$ It was postulated that this minority could be recognized by TL-tet, because TL-tet would only bind CD8 $\alpha \alpha$ homodimers. These cells would be selected 

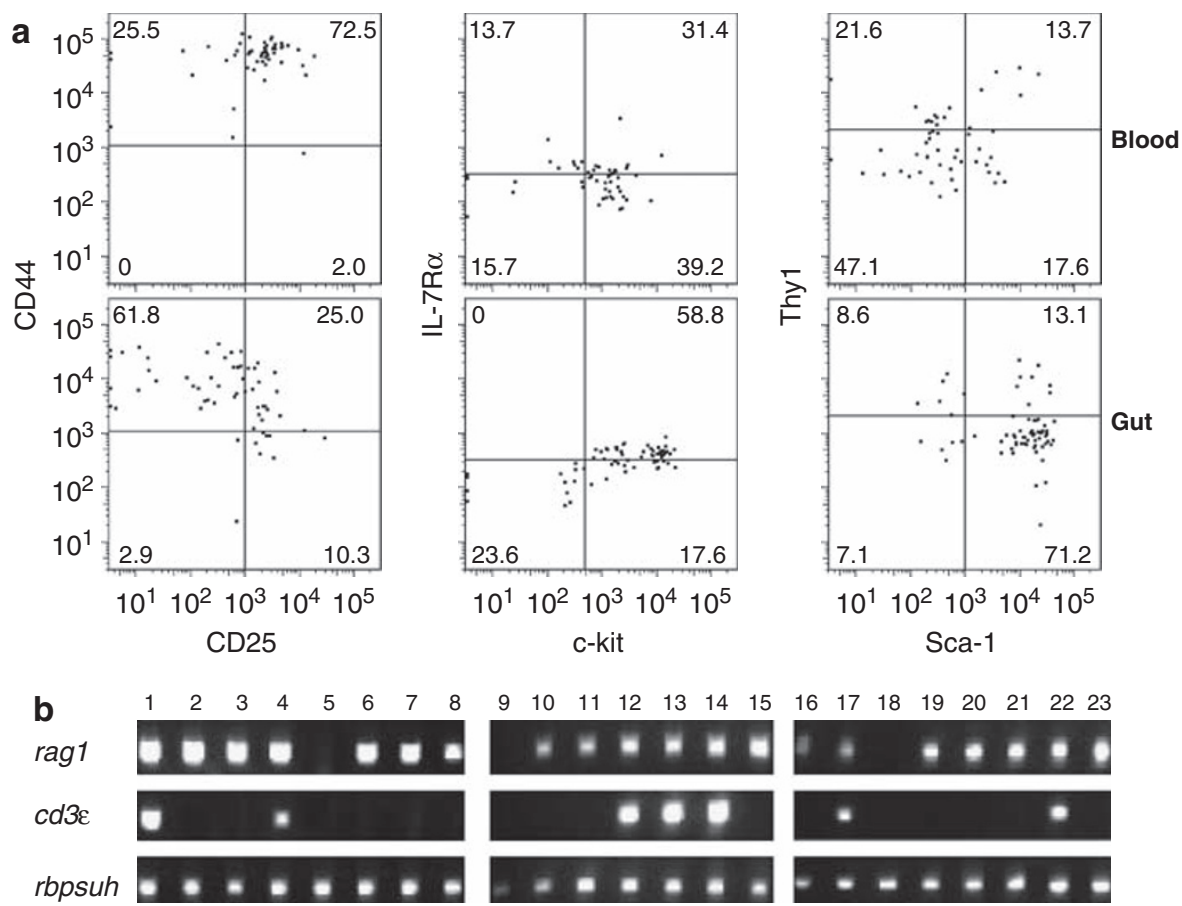

Figure 1 Characterization of triple negative populations able to egress the thymus. Thymus lobes from newborn CD45.2 T-cell antigen receptor (TCR)- $\alpha^{-/-}$mice were grafted into CD45.1 Rag- $2 / \gamma_{c}{ }^{-/-}$mice, and Lin ${ }^{-}$CD $45.2^{+}$cells of graft origin were studied in the blood, the gut lamina propria, and the spleen 10 days after transplant, as described in Supplementary Figure S2 online. (a) Phenotype of Lin- CD45.2 cells recovered from the blood and the gut lamina propria. Similar results were obtained in two experiments. (b) Lin- CD $45.2^{+}$cells were purified from the spleen, sorted as individual cells, and tested simultaneously for the expression of $c d 3 \varepsilon$ and rag1 mRNA. The simultaneous expression of $r b p s u h$ mRNA was tested to verify plating efficiency. Note: Although Lin- cells were clearly identified during analysis in all organs, the relative small blood volume that we could recover, the abundance of "nonspecific" contaminants in the blood and gut suspensions (platelets, bacteria, epithelial cells), and the rarity of precursors did not allow us to purify them from these organs.

by self-antigens in the thymus cortex, differentiate into agonist selected mature TCR- $\alpha \beta^{+}$DN cells that would then repopulate the TCR- $\alpha \beta$ CD $8 \alpha \alpha$ T-IEL. ${ }^{6}$ We first wished to characterize these putative DP CD8 $\alpha \alpha^{+}$precursors, but found that TL-tet did not discriminate $\mathrm{CD} 8 \alpha \alpha^{+}$cells in normal mice. The vast majority of CD $8 \alpha \beta^{+}$DP thymocytes and CD $8 \alpha \beta^{+}$T-IEL were TL-tet ${ }^{+}$, whereas an average of $15 \%$ of CD $8 \alpha \beta^{-}$CD $8 \alpha \alpha^{+}$T-IEL did not interact with these tetramers (Figure 3a). To further investigate the capacity of TL-tet to always recognize CD8 $\alpha \alpha$ complexes, we also studied $\mathrm{CD} 8^{+} \mathrm{T}$ cells from $\mathrm{CD} 8 \beta^{-}$-deficient mice that can only express CD8 $\alpha \alpha$ at the cell surface. As in normal mice, an average of $15 \%$ of $\mathrm{CD}^{+}{ }^{+}$T-IEL did not bind TL-tet (not shown) and the majority of peripheral $\mathrm{T}$ cells remained $\mathrm{TL}$-tet ${ }^{-} /$low (Figure 3a). We conclude that TL-tet does not allow the identification of cells expressing CD8 $\alpha \alpha$ homodimers. Therefore, it remains to be determined if any TL-tet ${ }^{+} \mathrm{DP}$ cells co-expresses $\mathrm{CD} 8 \alpha \alpha$, as the expression of these homodimers by DP cells was never directly investigated.

We next studied if TCR- $\alpha \beta^{+}$DN cells were agonist selected in normal conditions. We used mice expressing the endogenous mammary tumor virus (MMTV-Mls1 ${ }^{\mathrm{a}}$ ) that induces the deletion of self-reactive $\mathrm{V} \beta 6^{+}$cells in the thymus. We found that the bulk of NK1.1 ${ }^{-}$TCR- $\alpha \beta^{+}$DN thymocytes had undergone $\mathrm{V} 6^{+}$deletion (Figure $3 \mathbf{b}$ ). These results contrast to the agonist selected CD8 $\alpha \alpha$ T-IEL, in which V $\beta 6$-bearing cells are not deleted, ${ }^{23}$ and indicate that the vast majority of NK1.1 ${ }^{+}$ TCR- $\alpha \beta^{+}$DN thymocytes cannot originate CD $8 \alpha \alpha$ T-IEL.

\section{The relative role of TN and mature thymocyte sets in the generation of CD8 $\alpha \alpha$ T-IEL}

The above results did not exclude that part or even all CD $8 \alpha \alpha$ T-IEL would derive from a minority $\mathrm{TCR}^{+} \mathrm{DN}$ sub-population or from a yet other non-identified DP thymocyte set. To determine the relative contribution of the $\mathrm{TN}$ vs. the other more mature thymocytes in T-IEL generation, we compared T-IEL reconstitution after neonatal thymi transplantation in either Rag- $2^{-1-}$ or Rag- $2 / \gamma_{c}{ }^{-/-}$mice. We reasoned that CD8 $\alpha \alpha$ T-IEL deriving from early $\mathrm{TN}$ cells should be preferentially produced in Rag- $2 / \gamma_{c}{ }^{-1-}$ mice, whereas CD8 $\alpha \alpha$ T-IEL originating from more mature thymocytes (DP, conventional $\mathrm{CD} 4^{+} / \mathrm{CD} 8 \alpha \beta^{+}$, or mature $\mathrm{DN} \mathrm{TCR}^{+}$thymocytes) should be generated equally in either host (Figure 4a). Indeed, in Rag- $2 / \gamma_{c}{ }^{-1-}$ mice, the $\mathrm{CP}$ and immature local precursors are virtually absent. ${ }^{3}$ This empty niche should allow TN colonization. In contrast, very few TN precursors should be able to repopulate the full CP niche of the Rag-2-I- mice, which should hinder any new $\mathrm{TN}$ entry from the thymus. These mice were studied in detail in the first 3 weeks. As expected, the gut of both strains was equally colonized by conventional T cells $\left(\mathrm{CD} 4^{+}\right.$or CD $8 \alpha \beta^{+}$ T-IEL) 2 weeks after grafting (Figure 4b). Similarly, CD $8 \alpha \alpha$ 
$\%$ of S1p1-expressing cells

\begin{tabular}{lccccccc} 
& TN1 & TN1-2 & TN2 & TN3 & TN4 & DP & CD4 ${ }^{+}$SP \\
\hline Newborn & 22.5 & 42 & 17 & $\begin{array}{c}<2 \\
(3 / 240)\end{array}$ & $\begin{array}{c}<2 \\
(1 / 170)\end{array}$ & $\begin{array}{c}0 \\
(0 / 600)\end{array}$ & 51 \\
Adult & 18 & \multirow{2}{*}{36} & 19 & $\begin{array}{c}<2 \\
(2 / 220)\end{array}$ & $\begin{array}{c}<2 \\
(4 / 240)\end{array}$ & $\begin{array}{c}0 \\
(0 / 240)\end{array}$ & 44 \\
\hline
\end{tabular}

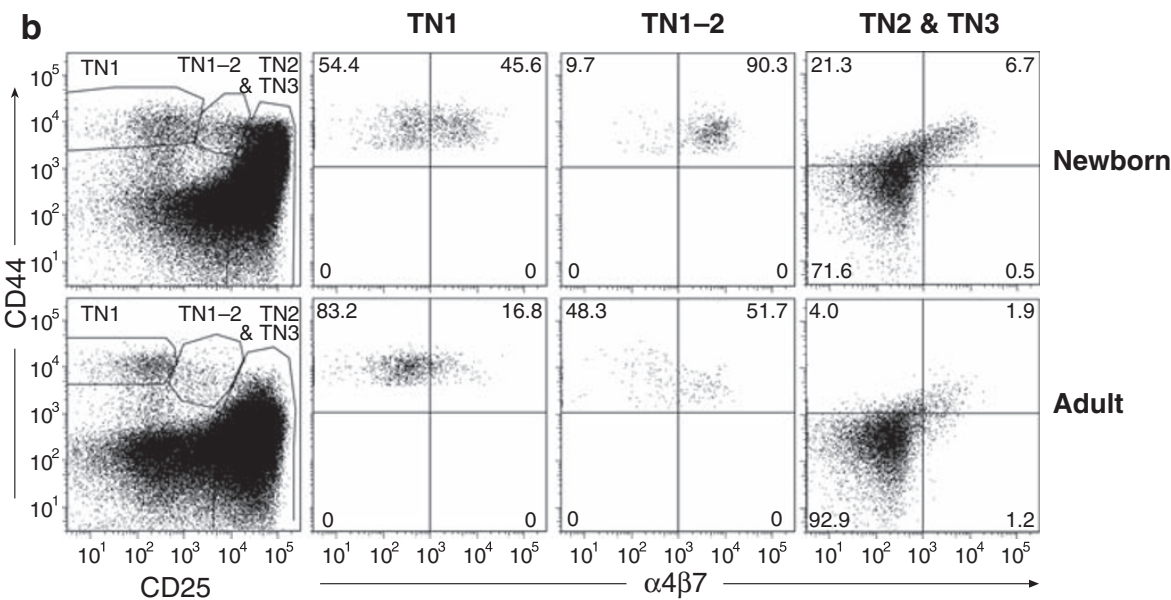

Figure 2 Expression of $\mathrm{S}_{1} \mathrm{P}_{1}$ and $\alpha 4 \beta 7$ during thymocyte development. (a) Results show the frequency of $s 1 p 1$ expression determined by single-cell reverse transcription-PCR in adult (6-8 weeks) or newborn (1-2 days) mice thymocyte sub-populations. The simultaneous expression of 28s mRNA was tested to verify plating efficiency. When frequency estimates were very low or $s 1 p 1$ was not detected, the number of positive vs. total cells studied is shown in parentheses. (b) Lin ${ }^{-}$thymocytes of adult or newborn mice were labeled with anti-CD44, anti-CD25, and anti- $\alpha 4 \beta 7$ monoclonal antibodies. Left: gates used to define different triple negative (TN) types. Right: $\alpha 4 \beta 7$ expression by different TN sub-populations. Data are representative of four experiments for adult mice and two experiments for newborn mice.

T-IEL were rapidly generated in Rag- $2 / \gamma_{c}{ }^{-/-}$mice (Figure $4 \mathbf{b}$ ), but the presence of immature precursors in the gut Rag- $2^{-/-}$ mice had a major impact on CD8 $\alpha \alpha$ T-IEL generation. Rather than reduced production we found a virtually complete block. Two weeks after transplantation, CD8 $\alpha \alpha$ T-IELs were barely detectable. By 3 weeks they were still very rare (Figure $4 \mathbf{b}$ ). Although a very minor contribution of other thymocyte sets cannot be formally excluded, these experiments show that reconstitution of this compartment that takes place in the first 3 weeks after birth depends on colonization of the gut by TN precursors and cannot be ensured by more mature thymocyte sets.

\section{DISCUSSION}

Although it is generally accepted that CD8 $\alpha \alpha$ T-IEL derive from precursor cells located in the gut $\mathrm{CP}$ in athymic mice, the role of a local T-differentiation process in normal conditions has been controversial. The reduced number of CD8 $\alpha \alpha$ T-IEL and the virtual absence of TCR- $\alpha \beta^{+}$cells in athymic mice, ${ }^{1}$ as well as the demonstration that these cells are thymus derived in normal mice, ${ }^{5}$ suggested that when the thymus was present, T-cell differentiation events in the gut might have little or no impact in CD8 $\alpha \alpha$ T-IEL generation. In contrast, the characterization of the thymus contribution to the CD8 $\alpha \alpha$ T-IEL pool that we describe here confers a fundamental role to the gut in local T cells' differentiation.
The thymus was believed to export only mature T cells, but our results identify another phase of thymus differentiation when immature thymocytes acquire the capacity to exit the thymus, circulate in the blood, and migrate to the gut. Indeed, we found that during the transition from the TN1 to the TN2 differentiation steps, thymocytes showed a major upregulation of the expression of the $\alpha_{4} \beta_{7}$ integrin, in particular, during the post-natal period, and that their S1p1 expression frequencies were similar to those found in mature thymocytes. The location of this cell set in the thymus could favor thymus egress, described to take place through capillaries located at cortex/medullary boundary. Indeed, TN1 cells are around this boundary and must cross this zone to reach the inner cortex, where TN2 cells reside. ${ }^{24}$ As TN1 to TN2 transition is accompanied by progressive CD25 upregulation, CD25int $\mathrm{TN} 1$ to TN2 transition cells might yet be near the cortex/medullary boundary and have a better access to the blood than their more mature counterparts. The study of $\mathrm{Lin}^{-}$precursors of thymus origin circulating in the blood confirmed that the bulk of the thymus egress occurs during the TN1 to TN2 transition, as the vast majority $\mathrm{Lin}^{-}$migrants recovered from the blood have the compound phenotype of CD $44^{+} \mathrm{CD} 25^{\text {int }}$ transitional cells, sharing their Thy1, Sca-1, IL-7R, and c-kit phenotypes. We also demonstrate directly that $\mathrm{Lin}^{-}$cells of thymus origin repopulate the gut LP. These cells are not just blood contaminants of gut suspensions, as the phenotype of recent thymus 

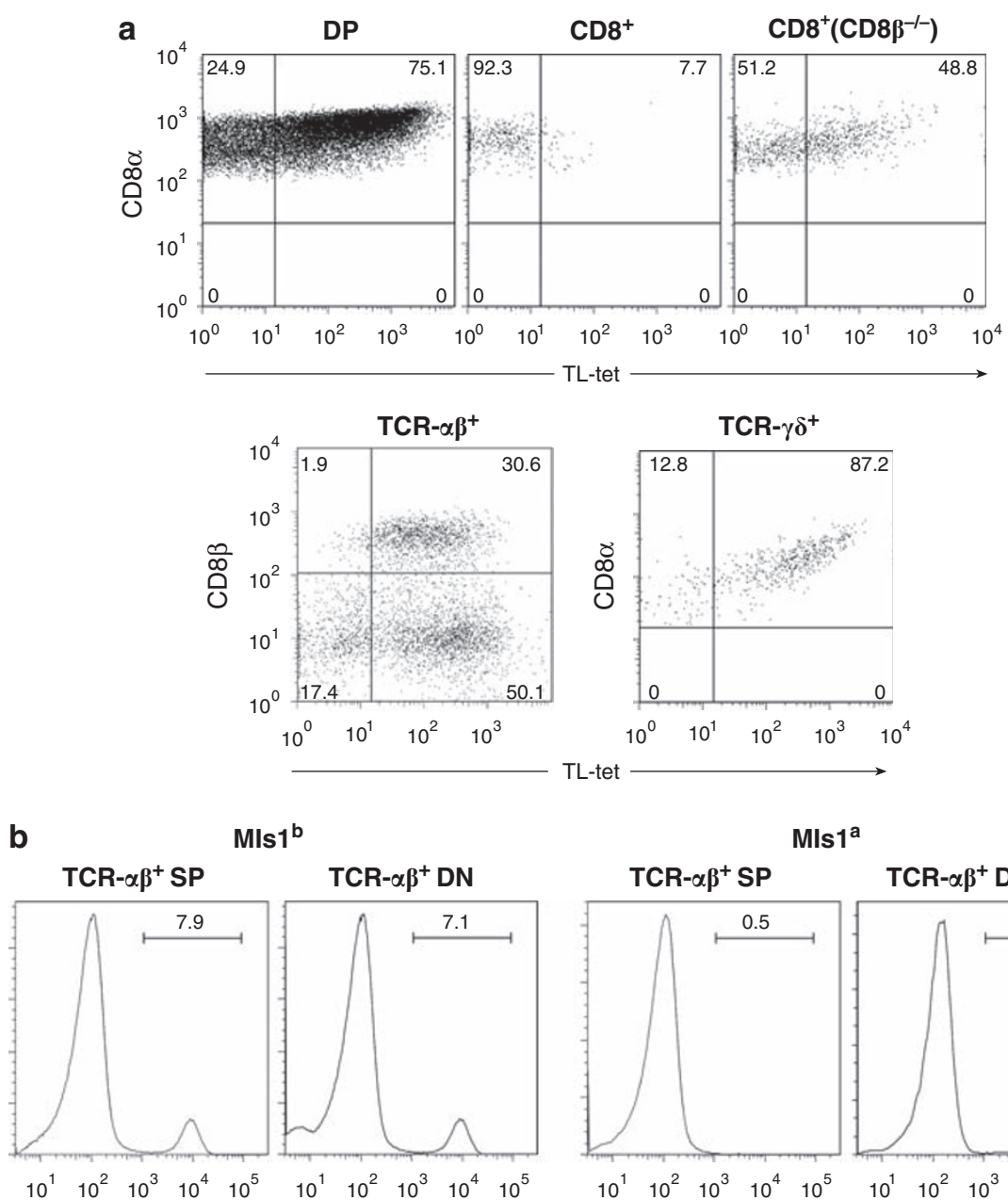

$M l s 1^{b}$ TCR- $\boldsymbol{\alpha} \boldsymbol{\beta}^{+} \mathrm{DN}$
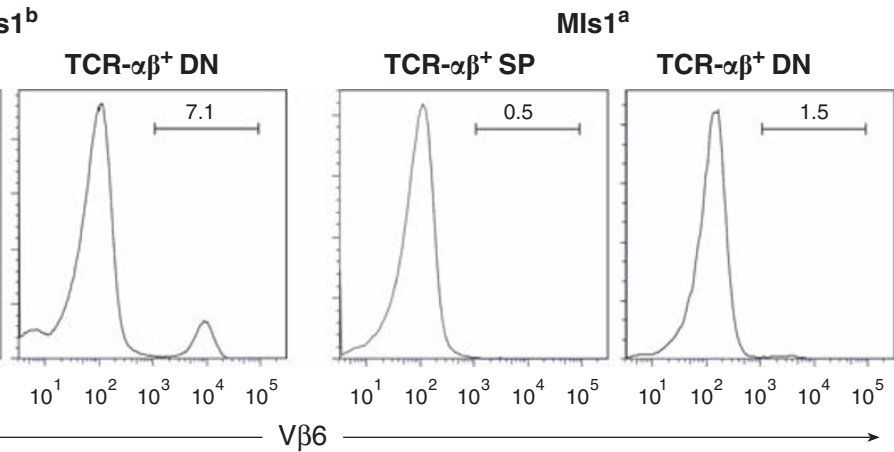

Figure 3 Thymus leukemia antigen-tetramers (TL-tet) binding and agonist selection in T cells. (a) TL-tet binding in CD8 ${ }^{+} \mathrm{T}$ cells. Upper graphs: left: $\mathrm{CD} 8 \alpha \beta^{+}$double-positive (DP) thymocytes; middle: $\mathrm{CD} 8 \alpha \beta^{+}$splenocytes; right: CD8 $\alpha \alpha^{+}$splenocytes from CD $8 \beta^{-/-}$mice. Lower graphs: intestinal CD8 $\alpha \alpha$ intraepithelial T lymphocytes (T-IELs). Left: T-cell antigen receptor (TCR)- $\alpha \beta^{+} ;$right: TCR- $\gamma \delta^{+}$. Cells from all organs were studied simultaneously. Data are representative of six experiments for normal mice and two experiments for CD8 $\beta^{-/-}$mice with the same results. (b) Frequency of $\mathrm{V} \beta 6^{+}$cells within TCR- $\alpha \beta^{+}$SP and NK1.1- TCR- $\alpha \beta^{+}$DN thymocytes from C57BI/6 (Mls $\left.1^{b}\right)$ and DBA/2 (Mls $1^{a}$ ) mice.

migrants in the blood and in the gut did not overlap. Both populations were $\mathrm{CD} 44^{+}$but virtually all cells recovered from the gut have acquired the characteristic phenotype of the Lin $^{-}$CP cells.

Although these data demonstrated that the thymus could export precursor cells to the gut, it was possible that these cells had a minor contribution to CD8 $\alpha \alpha$ T-IEL generation. Several independent experiments demonstrate that reconstitution of the CD8 $\alpha \alpha$ T-IEL compartment is a post-natal event, and that the thymus contribution to CD8 $\alpha \alpha$ T-IEL generation is restricted to the first 3 weeks after birth. At birth, IEL are virtually absent and only very rare TCR- $\gamma \delta$ cells are detected ${ }^{25} \mathrm{Lin}^{-} \mathrm{CP}$ cells are first dispersed in the LP and only start to aggregate around day 10 after birth. ${ }^{9}$ In neonatal thymectomized mice, CD8 $\alpha \alpha$ T-IEL are as found in nude mice, but when thymectomy is performed in 3-week-old mice, CD8 $\alpha \alpha$ T-IEL is not affected. ${ }^{5}$ Other studies indicate that after this period, the gut CD8 $\alpha \alpha$ T-IEL compartment is not renewed from outside. In thymus transplanted mice, we found that CPs of thymus origin maintained constant size and persisted until 5 months after transplant (the later point we studied), suggesting permanent colonization. ${ }^{11}$ Importantly, parabiosis experiments showed absence of CD8 $\alpha \alpha$ T-IEL chimerism between partners, demonstrating that the CD $8 \alpha \alpha$ T-IEL compartment is self-maintained in adult mice. ${ }^{4}$ In this context, our experiments to investigate the relative contribution of mature and immature thymocyte sets in transplanted mice were designed to focus this crucial 3 weeks reconstitution period, either mimicking the conditions found in the post-natal period (no precursor, no T cell as found in Rag- $2 / \gamma_{c}{ }^{-1-}$ mice) or the conditions allowing the migration of $\mathrm{TCR}^{+}$cells, but hindering precursor colonization (Rag-2 ${ }^{-1-}$ mice). We were surprised to observe that when precursors were prevented to colonize the gut, CD8 $\alpha \alpha$ T-IEL generation was virtually abrogated. Thus, when thymus lobes that can export both mature and immature thymocytes were transplanted into Rag- $2 / \gamma_{c}{ }^{-1-}$ mice both the conventional $\mathrm{CD} 4^{+} / \mathrm{CD} 8 \alpha \beta^{+}$and CD $8 \alpha \alpha$ T-IEL compartments 


\section{ARTICLES}

a

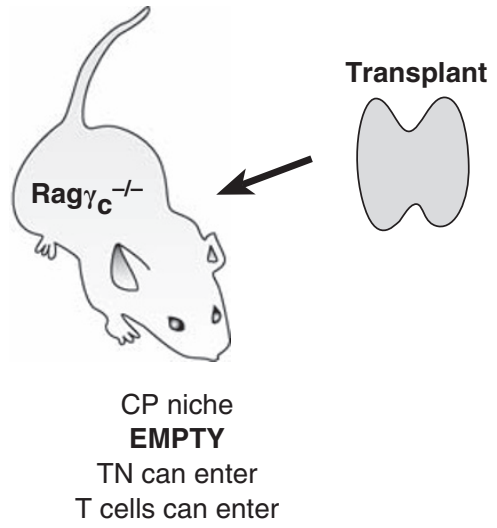

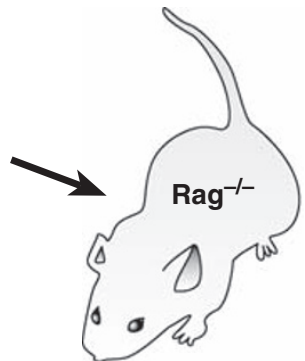

CP niche

FULL

TN cannot enter

$T$ cells can enter

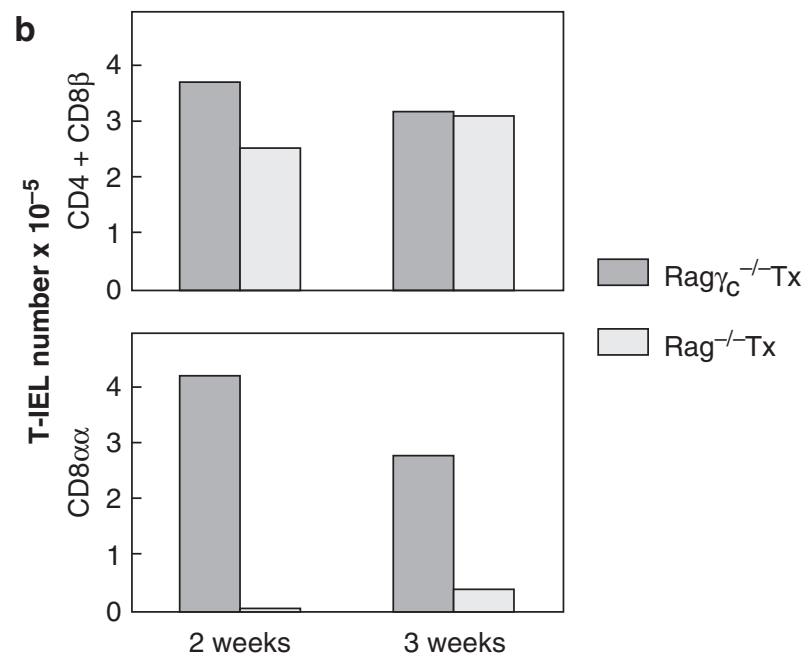

Total T-IEL

CD8 $\alpha \alpha$ T-IEL

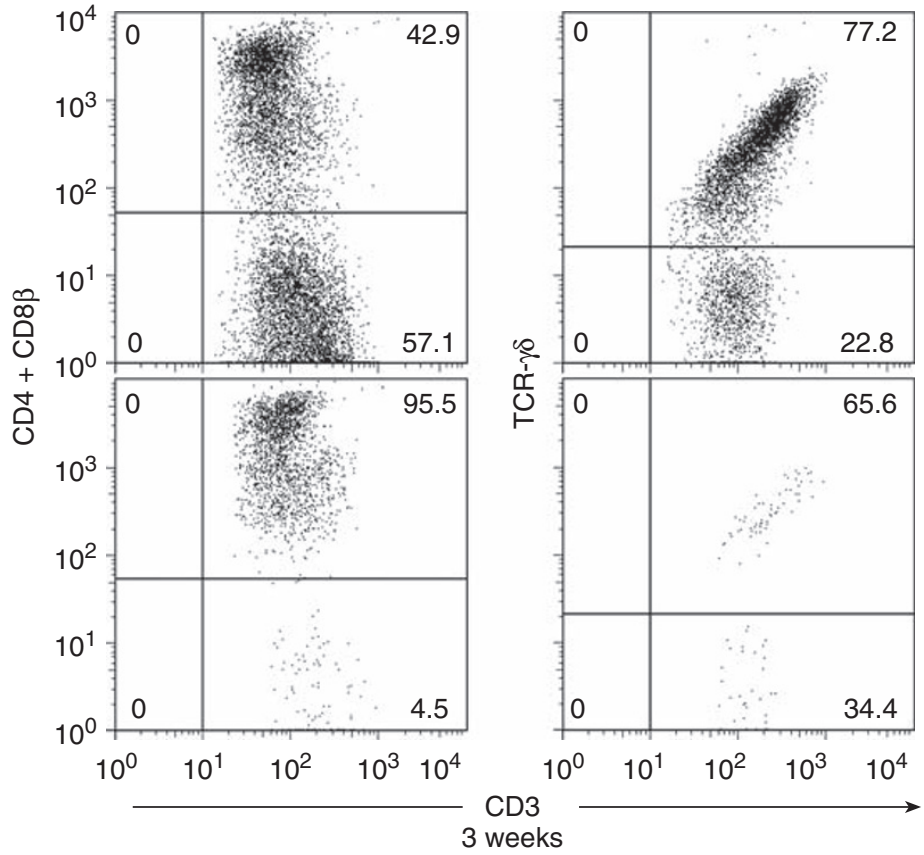

$\operatorname{Rag} \gamma_{c}{ }^{-1-} T x$

$\operatorname{Rag}^{-1-} \mathbf{T x}$

Figure 4 The relative role of triple negative (TN) cells and other thymocyte populations in CD8 $\alpha$ intraepithelial T lymphocytes (T-IEL) generation. Thymectomized CD45.1 Rag-2 ${ }^{-/-}$mice or CD45.1 Rag-2/ $\gamma_{c}{ }^{-/-}$mice were grafted with newborn CD45.2 thymi. (a) Schematic representation of the experimental strategy. (b): Upper graphs: numbers of CD45.2 $2^{+}$T-IEL studied at different time points after transplant; lower graphs: phenotype of CD45.2+ T-IEL 3 weeks after grafting. Note: CD8 $\alpha \alpha$ T-IEL were virtually absent in Rag-2 ${ }^{-/-}$mice by 2 weeks after thymus transplant. Data are from one full kinetics experiment out of two full kinetic studies with identical results. 
were rapidly reconstituted, demonstrating that the thymus is able to generate both cell types. In contrast, when thymus lobes were transplanted into Rag-2 $2^{-1-}$ mice, in which the gut immature precursor pool is present, only $\mathrm{CD} 4^{+} / \mathrm{CD} 8 \alpha \beta^{+}$were rapidly generated. During the 3 weeks period required for T-cell reconstitution, CD8 $\alpha \alpha$ T-IEL were virtually absent by 2 weeks and yet very rare by 3 weeks. It is possible that the rare CD8 $\alpha \alpha$ T-IEL generated in Rag- $2^{-1-}$ transplanted mice issue from more mature thymocytes. However, parabiosis experiments using Rag- $2^{-1-}$ mice demonstrated that their precursor pools are not fully impermeable, allowing the entry of rare precursors. ${ }^{26}$ Therefore, our results are fully compatible with the notion that the rare CD8 $\alpha \alpha$ T-IEL cells accumulating at late time points in Rag- $2^{-1-}$ transplanted mice issue from rare TN precursors yet able to enter their CP. Overall, these experiments demonstrate that CD8 $\alpha \alpha$ T-IEL in euthymic mice derive from very immature thymocyte precursors, the contribution of more mature thymocytes to their generation being very minor or null.

The identification of the $\mathrm{CD} 44^{+} \mathrm{CD} 25^{\text {int }} \mathrm{TN}$ population, as responsible for the generation of CD8 $\alpha \alpha$ T-IEL, allows to predict the properties of the thymus precursors colonizing the gut, and thus to discriminate thymus differentiation events from further T-cell differentiation steps occurring in the gut. TN1 cells have not initiated TCR rearrangements, and single cell cultures revealed that they have the capacity to generate both TCR- $\alpha \beta^{+}$ and TCR $-\gamma \delta^{+}$T cells. TCR rearrangements are yet rare in the TN2 set, and about $50 \%$ of TN2 thymocytes are yet bipotential. ${ }^{27}$ It is therefore likely that at least the majority of TN1 to TN2 cells have not yet rearranged their TCR and are bipotential, and that TCR rearrangements and TCR- $\alpha \beta$ and TCR- $\gamma \delta$ lineage commitment occurs in the gut. This notion is fully supported by other data. It was shown that transplants of Rag-deficient thymi reconstitute the gut $\mathrm{CP},{ }^{11}$ indicating that $\mathrm{CP}$ colonization may precede TCR rearrangements. Alike TN1 and TN2 sets, ${ }^{28}$ precursors located in the CP cells only express rare incomplete TCR- $\beta$ DJ rearrangements. They originate more mature precursor cells with TCR- $\alpha \beta$ and TCR- $\gamma \delta$ rearrangements located in the epithelium, ${ }^{12}$ where both $\beta$-selection ${ }^{9,12}$ and TCR- $\gamma \delta$ rearrangements ${ }^{29}$ were shown to occur. Finally, TN1 and TN2 thymocytes have yet the potential to generate NK (natural killer) cells. It was recently described that CP could be involved in the generation of a unique population of $\mathrm{NKp} 46^{+}$cells in the gut. ${ }^{10}$ It remains to be established if these cells also derive from TN precursors.

The present data contrast with previous reports proposing that CD8 $\alpha \alpha$ T-IEL would originate from a TL-tet ${ }^{+}$DP minority sharing with these IEL, the expression of CD8 $\alpha \alpha$. This cell set would be agonist selected in the thymus cortex, originating agonist selected TCR- $\alpha \beta^{+}$DN cells that would repopulate the gut. ${ }^{6}$ Our present data exclude this differentiation pathway. Thus, the expression of CD8 $\alpha \alpha$ homodimers in DP cells was never directly demonstrated, and we now show that TL-tet binding is not CD8 $\alpha \alpha$ specific, as these tetramers fail to bind many $\mathrm{CD} 8 \alpha \alpha^{+}$cells while adhering to several populations expressing $C D 8 \alpha \beta$. It was reported that binding of $C D 8 \alpha \beta^{+}$cells to tetramers of classic major histocompatibility complex class
I molecules is maximal in DP thymocytes and declines during differentiation into mature $\mathrm{CD} 8 \alpha \beta^{+} \mathrm{T}$ cells, and that such differential binding is determined by complex changes in the glycosylation status of the $\mathrm{CD} 8$ chains during ontogeny. ${ }^{30} \mathrm{We}$ found that TL-tet binding to CD8 complexes had very similar characteristics suggesting that CD8 glycosylation also has a major influence in TL binding. It must be noted that the notion that TL-tet recognizes CD8 $\alpha \alpha$ specifically results from binding studies performed with non-glycosylated CD $8 \alpha$ and $C D 8 \beta$ molecules. ${ }^{31}$ Our data show that it does not apply to the glycosylated CD8 molecules present in vivo. We also demonstrate that the vast majority of TCR- $\alpha \beta^{+} \mathrm{DN}$ cells is deleted of self-reactive cells, and thus cannot originate the self-reactive agonist selected CD8 $\alpha \alpha$ T-IEL pool.

To conclude, as the thymus and the gut epithelia share the same embryonic origin it was suggested that both would be able to sustain a T-cell differentiation process. The present results indicate that in the normal conditions the thymus is fundamental to ensure efficient T-cell commitment, as CD8 $\alpha \alpha$ T-IEL are rare in athymic mice. However, the gut must ensure all further T-cell differentiation steps, and thus has all characteristics of a primary lymphoid organ.

\section{METHODS}

Mice. DBA/2 mice were obtained from Charles River Laboratories (L'Abresle, France), CD45.2 mice from the Centre d'Elevage R Janvier (Le Genest St Isles, France), and CD45.1 mice from the Centre de Distribution, Typage et Archivage Animal (CDTA, Orléans, France). Nude (nu/nu) mice were from CDTA or Taconic (Denmark). CD45.1 Rag- $2^{-/-}$and CD45.1 Rag-2 ${ }^{-1-} \gamma_{c}{ }^{-1-}$ mice were a gift from JP Di Santo (Institut Pasteur, Paris, France). CD45.2 TCR- $\alpha^{-1-}$ mice were from A Lehuen (Hôpital Saint Vincent de Paul, Paris, France) and CD8 $\beta^{-/-}$ mice were a gift from A Singer (National Cancer Institute, Bethesda, MD).

Thymectomy, thymus transplants, and cell transfers. To completely remove the very small thymus rudiment of CD45.1 Rag-2 ${ }^{-/-}$and CD45.1 Rag- $2 / \gamma_{c}{ }^{-1-}$ mice, we use a special surgical procedure described elsewhere. ${ }^{32}$ Neonatal thymi were recovered $1-2$ days after birth using the same procedure, and one thymus lobe was transplanted under the kidney capsule of each recipient.

Cell isolation, antibodies, and TL-tet labeling. Intraepithelial and LP lymphocytes from the small intestine were obtained as described. ${ }^{12}$ For flow cytometry, cells were incubated with an anti-CD16/CD32 Fcreceptor monoclonal antibody (mAb) (2.4G2) to block Fc-mediated antibody binding, previous to cell surface staining. The following $\mathrm{mAbs}$ obtained from BD Pharmingen (San Diego, CA) were used: anti-CD3 (145-2C11), anti-CD8 $\alpha$ (53-6.7), anti-CD11b/Mac1 (M1/70), antiCD19 (1D3), anti-CD25 (PC-61), anti-CD45.1/Ly5.1 (A20-1.7), antiCD45.2/Ly5.2 (104-2.1), anti-CD90.2/Thy1.2 (53-2.1), anti-CD117/c-kit (2B8), anti-TCR $\beta$ (H57-597), anti-TCR $\delta$ (GL3), anti-Vß6 (44.22.1), anti-LPAM1/ $\alpha 4 \beta 7$ integrin (DATK/32), anti-NK1.1 (PK136), antiGR1 (8C5), anti-erythroid cells (TER-119), and anti-Sca-1 (E13-161.7). Anti-CD4 (GK1.5), anti-CD8 $\beta$ (H35-172), and anti-CD127/IL7-R $\alpha$ (A7R34, a gift from Dr S-I Nishikawa, Kyoto University and RIKEN Center, Kyoto, Japan) were purified and conjugated in our laboratory (Necker Institute, Paris, France). Anti-CD44 (1M781) was obtained from eBiosciences (San Diego, CA). All the above-mentioned mAbs were directly coupled to fluorescein isothiocyanate, phycoerythrin, 
PerCP-Cy5.5, PECy7, allophycocyanin, allophycocyanin-Alexa Fluor 750 , Pacific Blue, or conjugated with biotin. Biotinylated mAbs were revealed with PECy7-streptavidin (BD Pharmingen), phycoerythrin-Alexa Fluor 750-streptavidin (Invitrogen, Cammarillo, CA), Pacific Blue-streptavidin (Invitrogen) or Pacific Orange-streptavidin (Molecular Probes). Cells were analyzed on a FACSCalibur or a FACSCanto (Becton Dickinson, San Diego, CA). Phycoerythrin-conjugated TL-tet were obtained as described previously. ${ }^{33}$ We found that adequate TL-tet binding required particular approaches. Each lot had to be tested for maximal efficiency and TL-tet/cell number ratios should be strictly maintained. Moreover, any previous labeling with $\mathrm{mAbs}$ reduced considerably TL-tet staining. Therefore cells were first incubated with TL-tet for $1 \mathrm{~h}$ at $4^{\circ} \mathrm{C}$. Cells were then washed and incubated with the indicated antibodies.

For analysis and sorting of $\mathrm{CD} 4^{-} \mathrm{CD} 8^{-} \mathrm{CD} 3^{-}(\mathrm{TN})$ su-bpopulations, thymocytes were initially enriched for TN cells by depletion with beads coated with anti-CD8 $\alpha$ (Dynabeads M-450; Dynal AS, Oslo, Norway). Antibodies recognizing mature lineage-positive hematopoietic cells in all experiments were TER-119, NK1.1, GR1, Mac1, DX5, CD3, TCR- $\beta$, TCR- $\delta$, CD $8 \beta$, and CD19. Cells were sorted on a FACS ARIA (Becton Dickinson). The efficiency of purification was verified after each sorting and was higher than $99 \%$.

PCR analysis. Single cells were sorted by a FACS ARIA equipped with an automatic cell deposition unit (Becton Dickinson), and multiplex single-cell reverse transcription-PCR analysis was carried out as previously described. ${ }^{34}$ To allow evaluation of the expression of several mRNAs in the same cell, primers were selected to have the same efficiency, to amplify fragments of similar relatively small size and to provide amplifications where neither the primers nor the amplicons competed between themselves, and were the following: $c d 3 \varepsilon$ : 5' -TGGAACACTTTCTGGGGCAT-3', 5' -AAGTCTCCATCT CAGGAACC-3' (nested), and $3^{\prime}$ primer $5^{\prime}$-CTCCACACAGTACTCACA CA-3'; $\operatorname{rag} 1:$ 5': 5'-CAACCAAGCTGCAGACATTC-3'; 5'-GCAGACATTCTAGCACTCTG-3' (nested), and 3': 5' -CTACTGGAGACTGTTCTAGG-3'; rbpsuh 5' primers : 5' TGTGCATTGCTTCAGGAACG-3' 5' $^{\prime}$-GAAGGTGGCACTGTTCAA TC-3' (nested), and 3' primer 5' -GGTCGTCTGCATCCAGTAAT-3'; $28 s 5^{\prime}$ primer: $5^{\prime}$-TACCGGACCCTGAACAGAAT-3', and $3^{\prime}$ primer $5^{\prime}$-GTAGATCCTCCGGCATGTTT-3'; s1p1 primers were as described. ${ }^{35}$

SUPPLEMENTARY MATERIAL is linked to the online version of the paper at http://www.nature.com/mi

\section{ACKNOWLEDGMENTS}

We are grateful to J.P. Di Santo, A. Lehuhen, and A. Singer for providing mice, J.P. Di Santo, A. Freitas, and P. Vieira for reviewing this article, A. Legrand and F. Vasseur for technical help, and C. Bordin for mice breeding. A. Boudil is supported by a grant from the Ligue Nationale Contre le Cancer. L. Peaudecerf was initially supported by a grant from the Association pour la Recherche sur le Cancer, and is now supported by the European Research Council (ERC). This work was supported by the ERC.

\section{DISCLOSURE}

The authors declared no conflict of interest.

(C) 2011 Society for Mucosal Immunology

\section{REFERENCES}

1. Rocha, B. The extrathymic T-cell differentiation in the murine gut. Immunol. Rev. 215, 166-177 (2007).

2. Saito, H. et al. Generation of intestinal $T$ cells from progenitors residing in gut cryptopatches. Science 280, 275-278 (1998).

3. Kanamori, Y. et al. Identification of novel lymphoid tissues in murine intestinal mucosa where clusters of c-kit+ IL-7R+ Thy1+ lymphohemopoietic progenitors develop. J. Exp. Med. 184, 1449-1459 (1996).
4. Sugahara, S. et al. Extrathymic derivation of gut lymphocytes in parabiotic mice. Immunology 96, 57-65 (1999).

5. Lin, T., Matsuzaki, G., Kenai, H., Nakamura, T. \& Nomoto, K. Thymus influences the development of extrathymically derived intestinal intraepithelial lymphocytes. Eur. J. Immunol. 23, 1968-1974 (1993).

6. Gangadharan, D. et al. Identification of pre- and postselection TCRalphabeta+ intraepithelial lymphocyte precursors in the thymus. Immunity 25, 631-641 (2006).

7. Eberl, G. \& Littman, D.R. Thymic origin of intestinal alphabeta T cells revealed by fate mapping of RORgammat + cells. Science 305, 248-251 (2004).

8. Baldwin, T.A., Sandau, M.M., Jameson, S.C. \& Hogquist, K.A. The timing of TCR alpha expression critically influences T cell development and selection. J. Exp. Med. 202, 111-121 (2005).

9. Naito, T., Shiohara, T., Hibi, T., Suematsu, M. \& Ishikawa, H. ROR gamma $\mathrm{t}$ is dispensable for the development of intestinal mucosal T cells. Mucosal. Immunol. 1, 198-207 (2008).

10. Luci, C. et al. Influence of the transcription factor RORgammat on the development of NKp46+ cell populations in gut and skin. Nat. Immunol. 10, 75-82 (2009).

11. Lambolez, F. et al. The thymus exports long-lived fully committed T cell precursors that can colonize primary lymphoid organs. Nat. Immunol. 7 , 76-82 (2006).

12. Lambolez, F. et al. Characterization of T cell differentiation in the murine gut. J. Exp. Med. 195, 437-449 (2002).

13. Bas, A., Hammarstrom, S.G. \& Hammarstrom, M.L. Extrathymic TCR gene rearrangement in human small intestine: identification of new splice forms of recombination activating gene-1 mRNA with selective tissue expression. J. Immunol. 171, 3359-3371 (2003).

14. Bruno, L., Rocha, B., Rolink, A., von Boehmer, H. \& Rodewald, H.R. Intraand extra-thymic expression of the pre-T cell receptor alpha gene. Eur. J. Immunol. 25, 1877-1882 (1995).

15. Hamerman, J.A., Page, S.T. \& Pullen, A.M. Distinct methylation states of the CD8 beta gene in peripheral T cells and intraepithelial lymphocytes. J. Immunol. 159, 1240-1246 (1997).

16. Porritt, H.E. et al. Heterogeneity among DN1 prothymocytes reveals multiple progenitors with different capacities to generate T cell and non-T cell lineages. Immunity 20, 735-745 (2004).

17. Allman, D. et al. Thymopoiesis independent of common lymphoid progenitors. Nat. Immunol. 4, 168-174 (2003).

18. Suzuki, K. et al. Gut cryptopatches: direct evidence of extrathymic anatomical sites for intestinal T lymphopoiesis. Immunity 13, 691-702 (2000).

19. Matloubian, M. et al. Lymphocyte egress from thymus and peripheral lymphoid organs is dependent on S1P receptor 1. Nature 427, 355-360 (2004).

20. Wagner, N. et al. Critical role for beta7 integrins in formation of the gut-associated lymphoid tissue. Nature 382, 366-370 (1996).

21. Koseki, S. et al. In situ demonstration of intraepithelial lymphocyte adhesion to villus microvessels of the small intestine. Int. Immunol. 13, 1165-1174 (2001).

22. Rocha, B., von Boehmer, H. \& Guy-Grand, D. Selection of intraepithelial lymphocytes with CD8 alpha/alpha co-receptors by self-antigen in the murine gut. Proc. Natl. Acad. Sci. USA 89, 5336-5340 (1992).

23. Rocha, B., Vassalli, P. \& Guy-Grand, D. The V beta repertoire of mouse gut homodimeric alpha CD8+ intraepithelial T cell receptor alpha/ beta+lymphocytes reveals a major extrathymic pathway of T cell differentiation. J. Exp. Med. 173, 483-486 (1991).

24. Petrie, H.T. \& Zuniga-Pflucker, J.C. Zoned out: functional mapping of stromal signaling microenvironments in the thymus. Annu. Rev. Immunol. 25, 649-679 (2007).

25. Bandeira, A. et al. Extrathymic origin of intestinal intraepithelial lymphocytes bearing T-cell antigen receptor gamma delta. Proc. Natl. Acad. Sci. USA 88, 43-47 (1991).

26. Agenes, F., Rosado, M.M. \& Freitas, A.A. Independent homeostatic regulation of B cell compartments. Eur. J. Immunol. 27, 1801-1807 (1997).

27. Ciofani, M., Knowles, G.C., Wiest, D.L., von Boehmer, H. \& ZunigaPflucker, J.C. Stage-specific and differential notch dependency at the alphabeta and gammadelta T lineage bifurcation. Immunity 25, 105-116 (2006). 
28. Livak, F., Tourigny, M., Schatz, D.G. \& Petrie, H.T. Characterization of TCR gene rearrangements during adult murine T cell development. J. Immunol. 162, 2575-2580 (1999).

29. Laky, K. et al. Enterocyte expression of interleukin 7 induces development of gammadelta T cells and Peyer's patches. J. Exp. Med. 191, 15691580 (2000).

30. Daniels, M.A. et al. CD8 binding to MHC class I molecules is influenced by T cell maturation and glycosylation. Immunity 15, 1051-1061 (2001).

31. Leishman, A.J. et al. T cell responses modulated through interaction between CD8alphaalpha and the nonclassical MHC class I molecule, TL. Science 294, 1936-1939 (2001).
32. Lancrin, C. et al. Major T cell progenitor activity in bone marrow-derived spleen colonies. J. Exp. Med. 195, 919-929 (2002).

33. Pardigon, N., Darche, S., Kelsall, B., Bennink, J.R. \& Yewdell, J.W. The TL $\mathrm{MHC}$ class Ib molecule has only marginal effects on the activation, survival and trafficking of mouse small intestinal intraepithelial lymphocytes. Int. Immunol. 16, 1305-1313 (2004).

34. Peixoto, A., Monteiro, M., Rocha, B. \& Veiga-Fernandes, H. Quantification of multiple gene expression in individual cells. Genome. Res. 14, 1938-1947 (2004)

35. Carlson, C.M. et al. Kruppel-like factor 2 regulates thymocyte and T-cell migration. Nature 442, 299-302 (2006). 